\title{
Observing the Seyfert 2 nucleus of NGC 1068 with the VLT Interferometer
}

\author{
Huub Röttgering ${ }^{a}$, Walter Jaffe ${ }^{a}$, Klaus Meisenheimer ${ }^{b},{\text { Helen } \mathrm{Sol}^{c}, \text { Christoph Leinert }}^{b}$, \\ Andrea Richichi ${ }^{d}$ and Markus Wittkowski ${ }^{d}$ \\ ${ }^{a}$ Leiden Observatory, P.O. Box 9513, 2300 RA Leiden, The Netherlands; \\ ${ }^{b}$ Max-Planck-Institut für Astronomie, Königstuhl 17, D-69117 Heidelberg,Germany; \\ ${ }^{c}$ Observatoire de Paris-Meudon, 5 place J. Janssen, 92195 Meudon Cedex, France; \\ ${ }^{d}$ European Southern Observatory, Karl-Schwarzschild-Str. 2, D-85748 Garching bei München, \\ Germany.
}

\begin{abstract}
Dusty tori have been suggested to play a crucial role in determining the physical characteristics of active galactic nuclei (AGN), but investigation of their properties has stalled for lack of high resolution mid-IR imaging. Recently, a long-awaited breakthrough in this field was achieved: NGC 1068, a nearby AGN, was the first extragalactic object to be observed with a mid-IR interferometer, thereby obtaining the needed angular resolution to study the alleged torus. The instrument used was MIDI mounted on the ESO's VLT interferometer. The resulting $8-13 \mu \mathrm{m}$ interferometric spectra indicated the presence of a thick $(3 \times 4$ parsec $)$ configuration of warm dust surrounding a hot $\sim 1 \mathrm{pc}$ component, marginally elongated in the direction perpendicular to the main orientation of the warm component. The structure of the 10 micron "silicate" absorption feature hinted at the presence of non-typical dust.

In this proceeding, first the field of AGN research is briefly reviewed, with an emphasis on models of dusty tori. Second, the general properties of the key object NGC 1068 are discussed. Third, the MIDI data set is presented together with a first attempt to interpret this data in the context of tori models. Fourth, preliminary MIDI interferometric spectra of the nucleus of the nearby starbursting galaxy Circinus are presented. The apparent observed absence of both a hot component as well as a sharp absorption feature suggest that we view the torus more edge-on than is the case for NGC 1068. Finally, we briefly discuss the prospects of ESA's Darwin mission for observing nearby and distant AGN. The required capabilities for Darwin's first goal - the search for and subsequent characterization of earth-like planets orbiting nearby stars - are such that for its second goal - high resolution astrophysical imaging - the sensitivity will be similar to JWST and the angular resolution 1-2 orders better. This will allow detailed mapping of tori of low luminosity AGN such as NGC 1068 up to redshifts of 1 2 and more luminous AGN up to redshift of 10 and beyond.
\end{abstract}

Keywords: Galaxies: active; Infrared: general; Techniques: interferometric

\section{INTRODUCTION}

Active galactic nuclei reside in centers of galaxies and emit radiation over the entire electromagnetic spectrum, from the most energetic gamma-ray photons up to the longest radio waves. For a significant fraction of the AGN, the emission is so luminous that it outshines the entire associated galaxy. An intriguing fact is that AGN activity was much more common in the early Universe than it is today: there were a factor of 100 - 1000 more powerful AGN at $z \sim 1$ per co-moving volume than there are in our local universe. This suggests an intricate relation between the formation of galaxies and the activity of AGN. The hope is that a detailed study of the physics of AGN will lead to a deep understanding of this link.

The title of this conference is "advances in stellar interferometry". Contrary to what is suggested in this title, one of the main goals of the present rapid development in this field is to move to a situation where optical interferometry is a general tool in the study of many astrophysical phenomena. The VLT observatory with its 4 large telescopes has been especially designed with this goal in mind. An important step in making optical interferometry accessible for general astrophysical usage was the interferometric $8-13 \mu \mathrm{m}$ observations of the 
famous nearby Seyfert 2 galaxy NGC 1068, using the MIDI instrument mounted on the VLT Interferometer (VLTI). The results from these observations are reported in Jaffe et al (2004). Furthermore, the VINCI test camera operating in the K-band and also mounted on the VLTI turned out to have sufficient sensitivity to obtain fringes on NGC 1068 (see Wittkowski et al 2004). Finally, Swain et al (2003) have used the KECK interferometer to obtain visibility measurements on a northern Seyfert galaxy, NGC 4151. Swain reports on these results in this conference.

In this contribution, we will first briefly discuss some of the issues in the field of AGN, with an emphasis on the current status of models of dusty tori. Second, many different kinds of observational results on NGC 1068 are briefly touched upon. Third, the VLTI observations on NGC 1068 are presented and subsequently discussed in the context of models of dusty structures in the centers of active galaxies. Fourth, we briefly present preliminary results on MIDI observations of the Circinus galaxy, indicating that there may be a significant variety of structure of the dusty components in the hearts of AGN. Finally, we briefly discuss the potential of observations of distant AGN with ESA's space interferometer Darwin.

\section{ACTIVE GALACTIC NUCLEI}

With more and more observational techniques becoming available in the sixties and seventies of the last century, more and more classes of AGN were defined. This resulted in what is commonly referred to as the "zoo of AGN". This zoo contains may different types of objects including Seyfert 1 and 2, quasars, radio galaxies, emission line galaxies, BLACs, etc (e.g. Antonucci 1993, Urry \& Padovani 1995). An important aim of research into these objects is to understand the underlying physical processes that interrelate the various classes of AGN. In such studies a number of very important building blocks have either been found or suggested to be present. First, there is overwhelming evidence that the centers of AGN harbor massive black holes with masses ranging from $10^{6}$ up to $10^{9}$ solar masses. X-ray luminous accretion disks surrounds these black holes. Part of the material that is swirling in through such disks leaves this region though a collimated outflow, the jets. It has been suggested that the accretion disk is surrounded by a dusty structure, possibly in the form of a bagel: the dusty torus. Furthermore, characteristic components are two different types of emission line regions: the broad emission line regions with characteristic velocities of $10,000 \mathrm{~km} \mathrm{~s}^{-1}$ are thought to originate on spatial scales similar to the accretion disk $(\lesssim \mathrm{pc})$, while the narrow emission line region with velocities a factor $10-50$ smaller comes from scales encompassing that of the torus (few - $100 \mathrm{pc}$ ).

With all these building blocks a start can be made to attempt to relate the different classes of AGN. The mass of the central black hole has been suggested to be directly related to the maximum total energy output of the AGN, with the most luminous AGN harboring the most massive black holes. The amount of spin of the black hole possibly determines whether a well formed jet will emerge. A likely way to start the AGN activity seems to be the merging of two galaxies, which provides the material for the various AGN building blocks. This naturally leads to a time sequence from a building up phase, to a steady AGN, and finally the end of the activity. A very important role is thought to be played by the torus. Viewed edge-on, the line of sight towards the center is blocked, obscuring the nucleus and the broad line region. Viewed along the poles, the very luminous core and the broad line region are both apparent.

\section{MODELS OF DUSTY TORI}

With the existence of tori being an attractive way of understanding a number of observational characteristics of AGN, efforts were started to model the physical characteristics of the tori. Radiative transfer calculations were carried out for toroidal dusty structures surrounding an UV emitting source. The resulting IR spectrum can then be compared to what is observed. The first calculations were carried out by Pier and Krolik in 1992, followed by Efthathiou and Rowin-Robinson (1995) and Granato et al (1997). In general, a good match was found for the observed IR spectrum. Unfortunately no strong constraints could be placed on the general size and shape of the torus. However, at this time, this was not a major issue, since angular sizes of the tori were very much smaller than could be observed. Although this seemed to be a nice and consistent scenario, a number of unresolved problems remained. This was recently emphasized by Elitzur et al (2003) and the problems include: 
1. X-ray observations show large variations in X-ray column densities from object to object that are too large to be accounted for by simple uniform torus models.

2. Emission from normal dust in galactic or stellar environments often shows strong emission or absorption from the 10 micron silicate feature. The models need a considerable amount of fine-tuning to explain both the very modest features in Seyfert 2's and their absence in Seyfert 1's.

3. Some Seyfert galaxies have changed type, a behavior clearly not expected from the uniform torus models.

4. The stability of a uniform torus is an important issue: collisions within such a structure will quick lead to a disk that is substantially thinner than is required to explain the relative fraction of Seyfert 1's to Seyfert 2's.

As Krolik and Pier first noted and was recently elaborated on in Nenkova et al 2002, a number of these problems will disappear if a clumpy torus is introduced. This, however, significantly increases the number of parameters with which an obscuring torus can be described. It is therefore clear that the best way to establish the morphology of tori is to use high angular resolution observations.

\section{NGC 1068}

A prime target in the field of high resolution observation of the centers of AGN is NGC 1068. It is the closest and brightest in the IR of all AGN. With over 200 refereed papers containing its name in the title, it is the most studied AGN. Its nearly face-on orientation gives a beautiful view on its intricate spiral structure. A good point to start a discussion of the role of NGC 1068 in the understanding AGN physics is the important observation by Antonucci and Miller (1985). They showed that the polarized light of this narrow line object bears the signature of a broad line Seyfert 1 object. This naturally led to the conjecture of the presence of tori. The HST imaging of both narrow line and UV emission showed clear conical regions (Macchetto et al 1994). This was also interpreted in a scenario in which a torus permits only radiation from the nucleus to penetrate in two preferred and opposite directions. A next important high light was the observations of $\mathrm{H}_{2} 0$ maser emission (Greenhill et al 1996). Such emission originate in fairly warm $(400 \mathrm{~K})$ and dense $\left(10^{8}-10^{10} \mathrm{~cm}^{-3}\right)$ gas. In a velocity - position diagram, the measurements can be fitted by gas in a simple Keplerian orbit around a black hole. A more elaborate model by Lodato and Bertin (2003) suggests that the observed disk is self gravitating. They deduce for both the mass of the black hole and the disk $8 \times 10^{6} \mathrm{M}_{0}$. Sensitive VLBA measurements showed a pc-scale size disk interpreted as due to free-free emission from an ionized dense $\left(10^{6} \mathrm{~cm}^{-3}\right)$ and hot $\left(10^{7} \mathrm{~K}\right)$ gas (Gallimore et al 1997). Schinnerer et al 2000 concluded from their detailed mapping of the $12 \mathrm{CO}(2-1)$ line that there was evidence for a warped $100 \mathrm{pc} \mathrm{CO}$ emitting disk enclosing a mass of $10^{8} \mathrm{M}_{0}$. Finally, Chandra images revealed a detailed correspondence between the optical and X-ray morphologies, also indicating that the extended X-ray emission is beamed nuclear emission (Ogle et al 2003). The spectrum of the nucleus seems to be best fit by a power-law obscured by a Compton structure, possibly related to the torus (Matt et al 1997). Finally, deep images in the near and mid infrared have been taken by a number of authors (see for example Wittkowski et al 1998; Bock et al 2000; Gratadour et al 2003; Rouan et al 2004 and references therein). These images show a compact core with a number of addition morphological features within a region of 1 arcsec from this core. Most notably is the "tongue", a northern extension from the nucleus oriented in the same direction as the radio jet.

\section{VLT INTERFEROMETER}

Currently the VLT interferometer can make use of the four 8.2 meter VLT Unit Telescopes (UTs). Ultimately, when the $1.8 \mathrm{~m}$ Auxilary Telescopes (ATs) are installed the maximum attainable baseline is $2005 \mathrm{~m}$. A test camera by the name of VINCI can combine beams from two UTs and detect fringes in the K-band. The first science instrument, MIDI, currently observes in the wavelength region $8-13$ micron. For a detailed description we refer to Leinert et al (2003). An update to the 18-22 micron region is expected to become available in 2006. Finally, AMBER (see Petrov et al 2003) is also installed and operational at the VLTI and will be offered for general usage in 2005. This instrument will allow for simultaneous observations in the $\mathrm{J}, \mathrm{H}$, and $\mathrm{K}$ bands yielding in J-band a resolution of 1 mas for the maximum baseline of 205m. The sensitivity of AMBER and MIDI are such 
that tens of AGN should be observable. As discussed, torus models are not accurate enough to reliably predict the amplitude of the visibilities. Hence, there was the possibility that tori would be extended and smooth with low visibilities that would make it impossible to interferometrically observe nearby AGN with the first phase of VLTI instrumentation.

The potential of the VLTI to deliver high angular studies of AGNs will be further increased by two systems, FINITO and PRIMA. The former is already installed and under testing, the latter is to be available in 2006. FINITO is the external fringe tracker, which will permit integration times much longer than the atmospheric coherence time (hundredths of a second). PRIMA will permit differential phase measurements between a scientific target and a nearby reference star, achieving true imaging and astrometry at the ten-microarcsecond level. The resulting drastic increase in sensitivity would then certainly bring many AGN in the realm of IR interferometric observing.

\section{MIDI OBSERVATIONS OF NGC 1068}

As discussed, one of the brightest nearby AGN is NGC 1068. Our observations of this object were obtained during MIDI's initial scientific runs in June and November, 2003 and reported in Jaffe et al 2004. Figure 1a shows the corresponding $\mathrm{N}$-band spectrum of the source using the grism with a resolution $\mathrm{R}=30$, as integrated over the beam width of 0.4 arcsec. Fig. $1 \mathrm{~b}$ and $1 \mathrm{c}$ show the spectra as seen by the two-telescope VLT interferometer operating at the projected baselines: $B=42 \mathrm{~m}$ (resolution $\sim 50$ mas, orientated at a position angle $P . A . \simeq 40 \mathrm{deg}$ East of North), and $B=78 \mathrm{~m}$ (resolution $\sim 26$ mas, P.A. $=2$ deg i.e. almost the direction of the radio jet) respectively.

The simplest model, which well accounts for the spectra of both the total and interferometric flux in our measurements, requires only two Gaussian components of different temperature, size and foreground dust absorption $\tau_{S i O}$. The first is a hot $(T>800 \mathrm{~K})$, compact component for which we fix the temperature to nominally $1000 \mathrm{~K}$. Its length in the direction of the radio jet is well constrained to $1.1 \mathrm{pc}$; along the orthogonal direction we can set an upper limit $<0.9 \mathrm{pc}$. The second is a warm, well-resolved component $(3 \times 4 \mathrm{pc})$ with a best-fit temperature $T=270 \mathrm{~K}$. The $10 \mu \mathrm{m}$ absorption feature in the highest resolved interferometric observations (Fig. 1c) does not fit well to the profiles of common olivine-type silicate dust because those begin to drop already at $\sim 8 \mu \mathrm{m}$, while the interferometric spectrum does not seem affected shortward of $9 \mu \mathrm{m}$. Even if the observed profile might be modified by radiative transfer effects, we obtain a much better fit by using the profile of calcium aluminum-silicate $\left(\mathrm{Ca}_{2} \mathrm{Al}_{2} \mathrm{SiO}_{7}\right)$, a high temperature dust species found in some supergiant stars.

This simple heuristic model describes the basic observed scale sizes of the dust emission in NGC 1068. The torus models as described in section 3 can be divided in two classes: Compact tori (Pier and Krolik 1995), where the dust fills a thick cylinder (with an axial hole) of a few parsecs in diameter, and extended fat disk models (Efstathiou and Rowin-Robinson 1995; Granato et al 1997), with tens to hundreds of parsecs in diameter. Common to both classes was a very high absorption optical depth $\left(A_{V}>100\right.$, i.e. $\left.\tau_{12 \mu}>4\right)$ in the equatorial plane. This leads to the prediction that hot dust can only be observed from those surfaces which are both freely exposed to the radiation from the accretion disk and unobscured along the line-of-sight from the observer. In consequence, the observable structures at $270 \mathrm{~K}$ and at $1000 \mathrm{~K}$ are almost identical and suffer very similar dust absorption. Obviously, this is not what we observe in NGC 1068.

Already the unification between Seyfert 1 and 2 galaxies (Antonucci 1993) seems to demand a geometrically thick dust distribution, the height $h$ (above the midplane) of which should be similar to its radius $r$, i.e. $h / r \simeq 1$. By resolving the dust structure in NGC 1068 with MIDI/VLTI, we have demonstrated now that indeed $h / r \gtrsim 0.7$, even when allowing for projection effects (the axis of NGC 1068 is inclined by $\lesssim 20$ deg out of the plane of sky). Moreover, this thick structure is located at $r \leq 2 \mathrm{pc}$. Thus, irradiation by the nuclear source will produce a hot inner wall - a "funnel" which we identify as our hot component. Since the average cloud temperatures are low, $T<1000 \mathrm{~K}$, there is no way that gas pressure could support this structure against gravity in the nuclear potential of NGC 1068. Turbulent motions with average velocities $\left\langle v_{T}\right\rangle \simeq 100 \mathrm{~km} \mathrm{~s}^{-1}$ (i.e. similar to the random velocities which support the nuclear star cluster) have to prevent the cloud system from collapsing. However, collisions between those clouds are highly inelastic: The turbulent motion would be damped within about one orbital period, $t_{\text {orb }}(2 \mathrm{pc}) \simeq 10^{5}$ years. Thus, a continuous injection of kinetic energy into the cloud system seems 

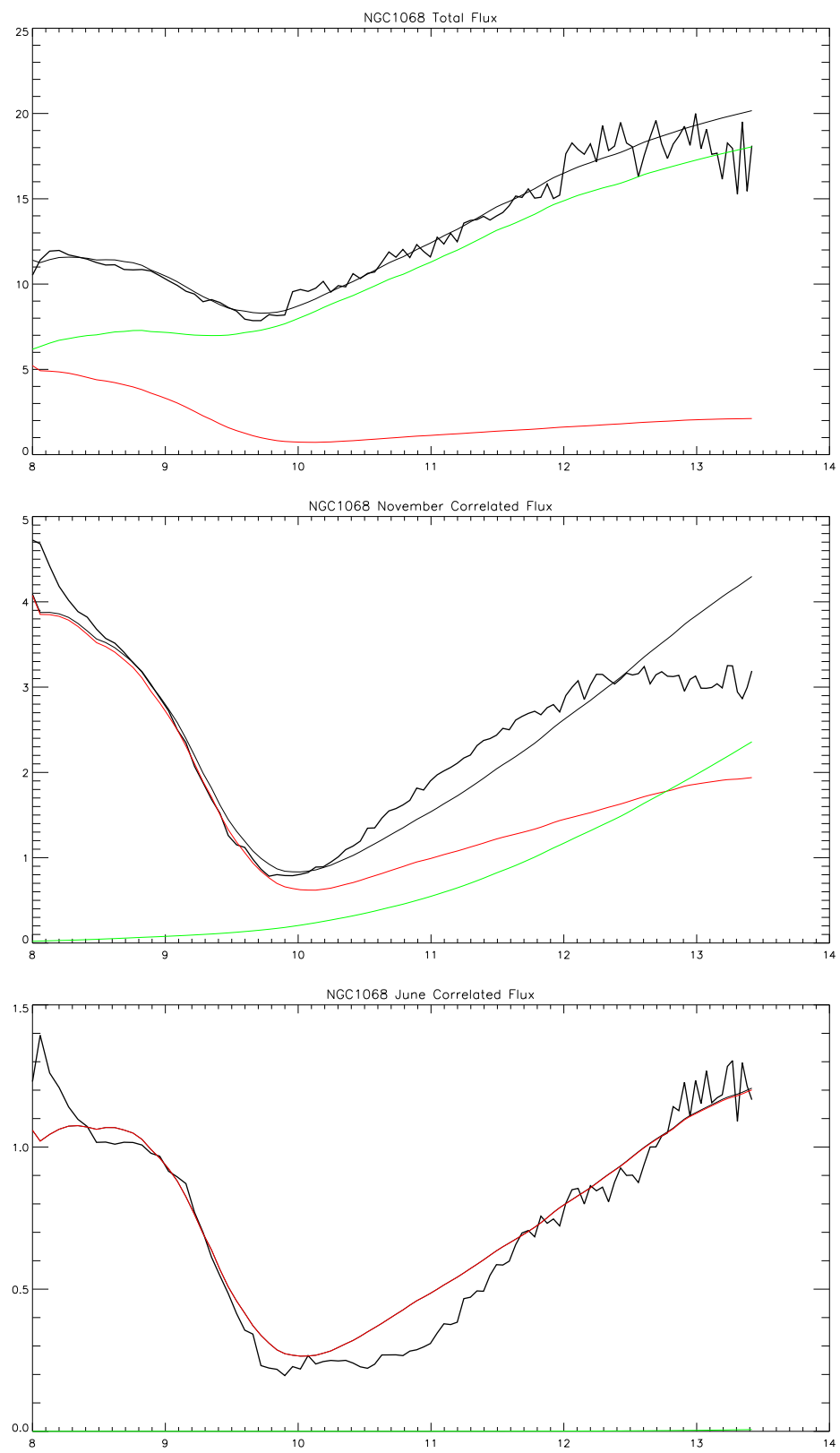

Figure 1. a. The single telescope, non-interferometric MIDI flux-density spectrum of the nucleus of NGC 1068. The jagged line shows the MIDI data and the smooth line the best fitting two-component Gaussian model described in the text. The upper dashed line shows the contribution of the warm component and the lower dashed line that of the hot component. The absorption dip near $10 \mu \mathrm{m}$ is caused primarily by 'astronomically common', olivine-type, silicate dust. b. The two-telescope interferometric spectrum from November 2003 with an effective resolution of $\sim 50$ mas. c. The interferometric spectrum from June 2003 with a resolution of $\sim 26$ mas. Here the dip near $10 \mu$ m is probably caused by dust of a an lumino-silicate or other, non-olivine, composition. (taken from Jaffe et al 2004).

to be required. To our knowledge, none of the current models of active galactic nuclei provide a convincing solution to this problem. Realistic torus models require both a physically motivated distribution of the dusty molecular clouds and a full 3-dimensional treatment of the radiative transfer. Thus, the first high resolution observations of the nucleus of an active galaxy by infrared interferometry forces a reconsideration of the physics 
of these spectacular objects.

\section{VINCI OBSERVATIONS OF NGC 1068}

The VINCI interferometric K-band observations of the core of NGC 1068 used a baseline of 46 meters and have been reported in Wittkowski et al 2004. The deduced squared visibility is $16 \%$. The simplest model consisted of a single Gaussian intensity distribution with a FWHM of 0.3 pc. Taking existing Speckle observations into account, a somewhat more complicated analysis constrained $40 \%$ of the flux to come from scales of clearly below $0.3 \mathrm{pc}$ and the remainder of the flux originating from scales smaller than the FOV, being $3.3 \mathrm{pc}$. The $0.3 \mathrm{pc}$ scale is to be rather similar to what Swain et al found for the core of NGC 4151. See also Swain's discussion during this meeting. With only one visibility point, it is clear that a discussion on the physical origin of such a compact structure is somewhat premature. Taking the suggested results of MIDI at face value, a likely possibility is that the compact VINCI component can be directly related to the $1000 \mathrm{~K}$ component, as observed with MIDI. The VINCI K-band spectrum would then originate from a hot funnel forming the inner boundary of the torus. Alternatively, the emission might come directly from the outer part of the hot accretion disk. Further observations that constrain for example the orientation of the structure will help distinguishing between these two possibilities.

\section{THE NEAR FUTURE}

Over the last three years we have been preparing a sample of 23 southern galaxies with a 10 micron flux density sufficiently bright to warrant MIDI interferometric observations (central flux densities $>400 \mathrm{mJy}$ ). All these sources have been observed at $10 \mu \mathrm{m}$ with the infrared imaging camera mounted on ESO's 3.6 m telescopes. These observations allowed us to measure the core flux on scales of 0.5 arcsec and obtain accurate positions. In addition, we are in the process of obtaining diffraction limited observations with NAOS-Conica in the HLM bands with the aim of deriving extinction maps, identifying outflow phenomena and determining the fraction of unresolved flux.

For the next three years, the goal of the MIDI program is two fold:

- An interferometric snapshot survey of all the sources from the entire list of targets. The plan is to measure two visibility points for each of two orthogonal baselines. This will deliver a good indication of the size of the thermal emission regions.

- A detailed interferometric study of a few selected targets. During 2004 and 2005 we will start this by obtaining further visibility points for NGC 1068. This will be used to investigate whether a simple toruslike morphology is a correct description or whether higher spatial frequency modifications are required (e.g., warps or clumpy tori).

In 2005-2006, VISIR can be used to take detailed spectra of the objects in this sample. This will allow us to observe the dust properties on scales of 0.5 arcsec, thereby addressing whether the dust properties change dramatically with distance from the nucleus as suggested by the NGC 1068 observations. This data will also be used to investigate the influence of a circumnuclear starburst on AGN activity.

At this time MIDI will be augmented with a 20-micron observing capability that we will use to map the outer, cooler regions of the torus. We hope to establish the connection between the warmer structures already observed and the larger (100-1000 pc) scale dust structures seen in many AGNs.

One step in this program was the observations of Circinus. This southern Seyfert 2 galaxy with a circum nuclear starburst has many characteristics that are distinctly different from NGC 1068. In Fig. 2 we show the preliminary reduction of the total and interferometric MIDI spectra taken in Feb 2004, using a projected baseline of $44 \mathrm{~m}$, yielding a resolution of $\sim 50$ mas.

Comparing the interferometric spectra of Circinus to that of NGC 1068, a number of differences are immediately apparent. First, the hot component that is strong at the shorter wavelengths for NGC 1068 seems virtually absent for Circinus. This might be due to orientation effects. If indeed the hot component in NGC 

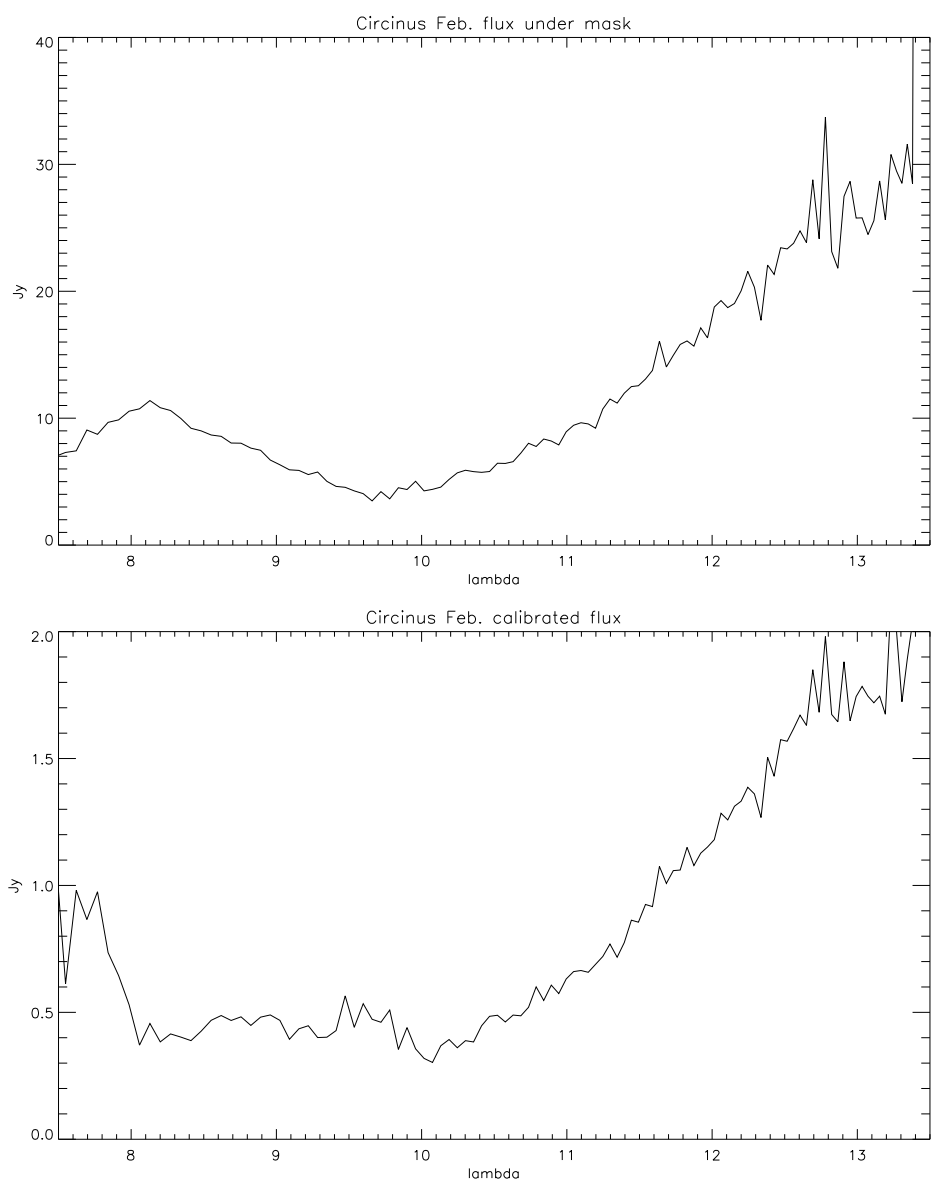

Figure 2. a. The single telescope, non-interferometric MIDI flux-density spectrum of the nucleus of CIRCINUS. b. The two-telescope interferometric spectrum with an effective resolution of $\sim 50$ mas.

1068 is due to a compact pc scale size structure within the torus, then such a structure could be hidden from our view if the torus were viewed more edge-on. This could be the case for Circinus where nuclear activity might be more hidden and the starburst more apparent. Second, the distinctly steep absorption, as seen in the NGC 1068 spectrum, is much less apparent for NGC 1068. This could also be due to orientation effects, if indeed the obscuring non-typical dust would be close to the pc scale structure which is hidden from our view.

\section{THE FUTURE: ESA'S DARWIN MISSION}

The proposed infrared space interferometry mission Darwin has two main aims: (i) to detect and characterize exo-planets similar to the Earth, and (ii) to carry out astrophysical imaging in the wavelength range $6-20 \mu \mathrm{m}$ at a sensitivity similar to JWST, but at an angular resolution up to 100 times higher. During this meeting Fridlund and Karlsson have discussed the Darwin mission and its use to find exo-earths. Here we will briefly discuss the performance of the Darwin imaging mission with an emphasis on the prospects for studying AGN (see also Röttgering et al 2003)

The present configuration for Darwin consists of 6 telescopes each with a diameter of $1.5 \mathrm{~m}$ and a central beam-combiner. Recent studies have shown that good performance can also be obtained in a very cost-effective way with fewer telescopes, each with a somewhat larger aperture. We again refer to the contributions of Fridlund and Karlsson during this meeting for a more detailed discussion of the trade-off involved. The relevant systems at the telescopes and the beam-combiner will be passively cooled to $40 \mathrm{~K}$, so that the sensitivity will be limited by shot-noise from emission by the zodiacal background (Fridlund et al 2001, Nakajima and Matshura 2001). 
This system should be a able to detect a point source of $2.5 \mu \mathrm{Jy}$ with a signal-to-noise of 5 in one hour of integration time. Such a sensitivity is comparable to that which is expected to be obtained with NASA's JWST mission. For sources that are more complex, assessing the integration time depends on the details of the morphology and the observing strategy (see Röttgering et al 2003). The maximum baselines that are foreseen are about 500 meter, which translates into a maximum resolution of 4 mas at 10 micron. This is a factor of more than 100 higher than the nominal resolution of 350 mas at 10 micron of the $6.4 \mathrm{~m}$ mirror of JWST.

While the telescopes are moving it is essential that the system is able to continuously measure the complex visibilities. To be able to do this, the array needs to remain co-phased while moving. Since in general the science targets are too faint to give enough signal for co-phasing, the light from an off-axis bright reference star will be used. A system based on such a principle is currently being implemented at the VLTI under the name PRIMA, which stands for Phase-Referenced Imaging and Microarcsecond Astrometry (e.g. Paresce et al 2003).

With these sensitivities and resolution, it is very clear that Darwin will be able to map nearby tori in exquisite detail. An interesting question is to what distance the tori of AGN can be mapped. To investigate this, we will use the torus models of Granato et al 1997 and results as presented in Röttgering et al 2003. In these models, the inner radii of these tori, $r_{\text {in }}$, are set by the distance from the central source at which the dust grains sublimate due to strong nuclear radiation. This radius is larger for more luminous AGN. For the models of Granato et al, $r_{\text {in }} \sim 0.5 L_{46}^{1 / 2} \mathrm{pc}$, where $L_{46}$ is the luminosity of the central optical UV emitter in units of $10^{46} \mathrm{ergs} \mathrm{s}^{-1}$. As a scale size of the torus D we will use $300 r_{\text {in }}$. In Figure 3 the diameter D and the 10 micron luminosity (which are now directly coupled) are given as a function of $z$ for angular scales of $1,0.1$ and 0.01 arcsec. The dotted lines correspond to the 10 micron luminosities as a function of $z$ for 10 micron flux densities of 5 and $50 \mu \mathrm{Jy}$.

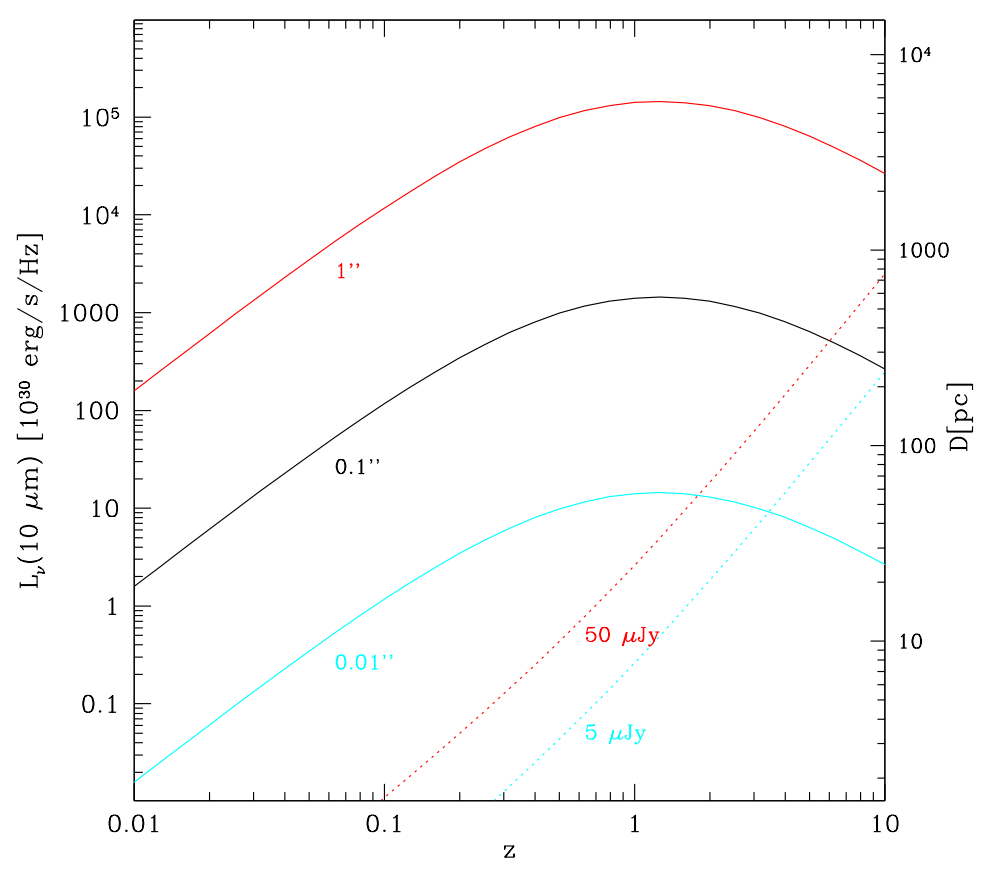

Figure 3. The 10 micron luminosity and physical scale D of dusty tori as a function of redshift. The solid lines are for observed angular scales of $1,0.1$ and 0.01 arcsec. The dotted lines are for an observed 10 micron flux density of 5 and 50 $\mu \mathrm{Jy}$. The computations have been done for $H_{0}=75 \mathrm{~km} \mathrm{~s}^{-1} \mathrm{Mpc}^{-1}$ and $\Omega=1$ (from Röttgering et al 2003).

A relatively weak AGN such as NGC 1068 has a 10 micron luminosity on the order of $1.7 \times 10^{31} \mathrm{erg} \mathrm{s}^{-1}$ $\mathrm{Hz}^{-1}$ and its modeled torus size is $60 \mathrm{pc}$. Note that the MIDI observations indicate that tori are likely to be 
significantly smaller. Up to redshifts of $z=1-2$ such weak AGN are bright enough to make a map with a ratio of total signal over noise per angular resolution element of more than 50. Also Darwin's resolution is very adequate for imaging the tori at these redshifts. Brighter AGN can basically be mapped up to a redshift of $z=10-20$ (if they exist).

This shows that Darwin cannot only study the physics of dusty tori in our local universe, but also at large redshifts. This will give the unique opportunity to investigate how the properties of tori change with redshift and when and how these tori and their associated massive black holes are built up at an epoch when galaxies are forming.

\section{REFERENCES}

1. W. Jaffe, K. Meisenheimer, H. J. A. Röttgering, C. Leinert, A. Richichi, O. Chesneau, D. Fraix-Burnet, A. Glazenborg-Kluttig, G.-L. Granato, U. Graser, B. Heijligers, R. Köhler, F. Malbet, G. K. Miley, F. Paresce, J.-W. Pel, G. Perrin, F. Przygodda, M. Schoeller, H. Sol, L. B. F. M. Waters, G. Weigelt, J. Woillez, and P. T. de Zeeuw, "The central dusty torus in the active nucleus of NGC 1068," Nature 429, pp. 47-49, May 2004.

2. M. Wittkowski, P. Kervella, R. Arsenault, F. Paresce, T. Beckert, and G. Weigelt, "VLTI/VINCI observations of the nucleus of NGC 1068 using the adaptive optics system MACAO," AEAA 418, pp. L39-L42, Apr. 2004.

3. M. Swain, G. Vasisht, R. Akeson, J. Monnier, R. Millan-Gabet, E. Serabyn, M. Creech-Eakman, G. van Belle, J. Beletic, C. Beichman, A. Boden, A. Booth, M. Colavita, J. Gathright, M. Hrynevych, C. Koresko, D. Le Mignant, R. Ligon, B. Mennesson, C. Neyman, A. Sargent, M. Shao, R. Thompson, S. Unwin, and P. Wizinowich, "Interferometer Observations of Subparsec-Scale Infrared Emission in the Nucleus of NGC 4151," ApJ 596, pp. L163-L166, Oct. 2003.

4. R. Antonucci, "Unified models for active galactic nuclei and quasars," ARAEA 31, pp. 473-521, 1993.

5. C. M. Urry and P. Padovani, "Unified Schemes for Radio-Loud Active Galactic Nuclei," PASP 107, p. 803, Sept. 1995.

6. E. A. Pier and J. H. Krolik, "Infrared spectra of obscuring dust tori around active galactic nuclei. I Calculational method and basic trends," ApJ 401, p. 99, Dec. 1992.

7. A. Efstathiou and M. Rowan-Robinson, "Dusty discs in active galactic nuclei," MNRAS 273, pp. 649-661, Apr. 1995.

8. G. Granato, L. Danese, and A. Franceschini, "Thick Tori around Active Galactic Nuclei: The Case for Extended Tori and Consequences for Their X-Ray and Infrared Emission," ApJ 486, p. 147, Sept. 1997.

9. M. Elitzur, M. Nenkova, and Z. Ivezic, "IR emission from AGNs," ArXiv Astrophysics e-prints, Sept. 2003.

10. M. Nenkova, Ž. Ivezić, and M. Elitzur, "Dust Emission from Active Galactic Nuclei," ApJ 570, pp. L9-L12, May 2002.

11. R. R. J. Antonucci and J. S. Miller, "Spectropolarimetry and the nature of NGC 1068," ApJ 297, pp. 621632, Oct. 1985.

12. F. Macchetto, A. Capetti, W. B. Sparks, D. J. Axon, and A. Boksenberg, "HST/FOC imaging of the narrow-line region of NGC 1068," ApJ 435, pp. L15-L18, Nov. 1994.

13. L. J. Greenhill, C. R. Gwinn, R. Antonucci, and R. Barvainis, "VLBI Imaging of Water Maser Emission from the Nuclear Torus of NGC 1068," ApJ 472, pp. L21+, Nov. 1996.

14. G. Lodato and G. Bertin, "Non-Keplerian rotation in the nucleus of NGC 1068 Evidence for a massive accretion disk?," A\&3A 398, pp. 517-524, Feb. 2003.

15. J. F. Gallimore, S. A. Baum, and C. P. O'Dea, "A direct image of the obscuring disk surrounding an active galactic nucleus.," Nature 388, pp. 852-854, 1997.

16. E. Schinnerer, A. Eckart, L. J. Tacconi, R. Genzel, and D. Downes, "Bars and Warps Traced by the Molecular Gas in the Seyfert 2 Galaxy NGC 1068," ApJ 533, pp. 850-868, Apr. 2000.

17. P. M. Ogle, T. Brookings, C. R. Canizares, J. C. Lee, and H. L. Marshall, "Testing the Seyfert unification theory: Chandra HETGS observations of NGC 1068," A\&̇A 402, pp. 849-864, May 2003. 
18. G. Matt, M. Guainazzi, F. Frontera, L. Bassani, W. N. Brandt, A. C. Fabian, F. Fiore, F. Haardt, K. Iwasawa, R. Maiolino, G. Malaguti, A. Marconi, A. Matteuzzi, S. Molendi, G. C. Perola, S. Piraino, and L. Piro, "Hard X-ray detection of NGC 1068 with BeppoSAX.," A 6 A 325, pp. L13-L16, Sept. 1997.

19. M. Wittkowski, Y. Balega, T. Beckert, W. J. Duschl, K.-H. Hofmann, and G. Weigelt, "Diffraction-limited 76 mas speckle masking observations of the core of NGC 1068 with the SAO $6 \mathrm{~m}$ telescope," $A \& A \mathbf{3 2 9}$, pp. L45-L48, Jan. 1998.

20. J. J. Bock, G. Neugebauer, K. Matthews, B. T. Soifer, E. E. Becklin, M. Ressler, K. Marsh, M. W. Werner, E. Egami, and R. Blandford, "High Spatial Resolution Imaging of NGC 1068 in the Mid-Infrared," $A J \mathbf{1 2 0 ,}$ pp. 2904-2919, Dec. 2000.

21. D. Gratadour, Y. Clénet, D. Rouan, O. Lai, and T. Forveille, "High angular resolution K-band spectroscopy of the nucleus of NGC 1068 with PUEO-GRIF (CFHT)," A\&A 411, pp. 335-342, Dec. 2003.

22. D. Rouan, F. Lacombe, E. Gendron, D. Gratadour, Y. Clénet, A.-M. Lagrange, D. Mouillet, C. Boisson, G. Rousset, T. Fusco, L. Mugnier, M. Séchaud, N. Thatte, R. Genzel, P. Gigan, R. Arsenault, and P. Kern, "Hot Very Small dust Grains in NGC 1068 seen in jet induced structures thanks to VLT/NACO adaptive optics," A 6 A 417, pp. L1-L4, Apr. 2004.

23. C. Leinert, U. Graser, A. Richichi, M. Schöller, L. F. B. M. Waters, G. Perrin, W. Jaffe, B. Lopez, A. Glazenborg-Kluttig, F. Przygodda, S. Morel, P. Biereichel, N. Haddad, N. Housen, and A. Wallander, "MIDI combines light from the VLTI: the start of $10 \mu \mathrm{m}$ interferometry at ESO," The Messenger $\mathbf{1 1 2}$, pp. 13-+, June 2003.

24. R. G. Petrov, F. Malbet, G. Weigelt, F. Lisi, P. Puget, P. Antonelli, U. Beckmann, S. Lagarde, E. Lecoarer, S. Robbe-Dubois, G. Duvert, S. Gennari, A. Chelli, M. Dugue, K. Rousselet-Perraut, M. Vannier, and D. Mourard, "Using the near infrared VLTI instrument AMBER," in Interferometry for Optical Astronomy II. Edited by Wesley A. Traub. Proceedings of the SPIE, Volume 4838, pp. 924-933 (2003)., pp. 924-933, Feb. 2003.

25. M. Fridlund et al., 2000. "Darwin, the Infrared Space Interferometer, Concepts and Feasibility Study Report, ESA report ESA-SCI, 2000, 12".

26. T. Nakajima and H. Matsuhara, "Sensitivity of an Imaging Space Infrared Interferometer," Appl. Opt. 40, pp. 514-526, Feb. 2001.

27. H. J. A. Röttgering, L. D’Arcio, C. Eiroa, I. Labbé, and G. Rudnick, "Astrophysical imaging with the Darwin IR interferometer," in ESA SP-539: Earths: DARWIN/TPF and the Search for Extrasolar Terrestrial Planets, pp. 299-+, Oct. 2003.

28. F. Paresce, F. Delplancke, F. Derie, A. Glindemann, A. Richichi, and M. Tarenghi, "Scientific objectives of ESO's PRIMA facility," in Interferometry for Optical Astronomy II. Edited by Wesley A. Traub. Proceedings of the SPIE, Volume 4838, pp. 486-495 (2003)., pp. 486-495, Feb. 2003. 